\title{
Influence of Temperature on the Ultrasonic Degradation of Poly(vinyl acetate) and Poly(vinyl chloride)
}

\author{
Sujay Chattopadhyay, Giridhar Madras \\ Department of Chemical Engineering, Indian Institute of Science, Bangalore- 560012, India
}

Received 7 February 2002; accepted 1 October 2002

\begin{abstract}
This study investigates the effect of temperature on the ultrasonic degradation of polyvinyl acetate and polyvinyl chloride in chlorobenzene. The time evolution of molecular weight distribution was determined using gel permeation chromatography. Continuous distribution kinetics was used to obtain the degradation rate coefficients. The rate coefficients decrease with increasing temperature, and this is attributed to the increase of vapor pressure and the
\end{abstract}

decrease of kinematic viscosity. The degradation rate coefficient also changes sharply near the glass transition temperature, indicating that this factor may play a role in the degradation process. (c) 2003 Wiley Periodicals, Inc. J Appl Polym Sci 88: 2818-2822, 2003

Key words: degradation; gel permeation chromatography; glass transition; viscosity; effect of temperature

\section{INTRODUCTION}

Ultrasound has been used in a variety of applications like homo- and copolymerization, ${ }^{1}$ emulsification, ${ }^{2}$ and organic reactions. ${ }^{3}$ One of the main applications of ultrasound has been in the area of polymer degradation. The literature has been summarized in the review articles by Basedow ${ }^{4}$ and Price. ${ }^{5}$ The study of ultrasound initiated degradation is interesting from both practical and academic viewpoints. ${ }^{6}$

Ultrasonic depolymerization is mainly caused by the mechanical forces that arise due to the propagation of ultrasonic waves. These forces are directly associated with cavitation, which is the nucleation, growth, and collapse of vapor-filled bubbles in the liquid. ${ }^{7}$ Microjets, formed during the collapse of a bubble, transmits the shear force on to the polymer chain. ${ }^{8}$ Unlike photo or thermal, the process of chain breakage is nonrandom and happens specifically at nearly the mid point of the chain. An explanation put forth by Thomas ${ }^{8}$ is that the polymer molecule gradually changes its shape (in a shear field) from that of a sphere to a cylinder and then this breaks at the midpoint.

Several investigations ${ }^{9,10}$ have focused on the physics of shear breakage and the factors that influence the cavitation. Other than the solvent polymer parameters like the Flory-Huggins and the Huggins constant that play a minor role, the main parameters are the vapor pressure and kinematic viscosity of the solution. ${ }^{11}$ Unlike thermal degradation, an increase of temperature leads to reduced

Correspondence to: G. Madras (giridhar@chemeng.iisc. ernet.in).

Journal of Applied Polymer Science, Vol. 88, 2818-2822 (2003) (C) 2003 Wiley Periodicals, Inc. rate coefficients. Price ${ }^{5}$ cites the work of Schmid and Beuttenmuller, who investigated the degradation of nitrocellulose dissolved in $n$-butyl acetate and of polystyrene in toluene at $40-120^{\circ} \mathrm{C}$. The results indicated that the degradation proceeded more slowly and yielded higher limiting molecular weights at higher temperatures. Thomas and Alexander ${ }^{12}$ investigated ultrasonic degradation of cellulose nitrate in a series of alkyl acetates from 0 to $85^{\circ} \mathrm{C}$ and observed an optimum temperature. The reduced degradation observed at both higher and lower temperatures was explained by ineffective cavitation. Malhotra ${ }^{13}$ investigated ultrasonic degradation of hydroxypropyl cellulose in water, ethanol, and tetrahydrofuran at various temperatures. It was concluded that for cavitation to occur there must be a critical radius that is dependent on the wavelength and intensity of sound, density, viscosity, and surface tension of the solvent. Recently, Price ${ }^{14}$ investigated the degradation of polystyrene in toluene also reported decreasing rate coefficients with increasing temperature.

To our knowledge, we are unaware of any study on the ultrasonic degradation of polyvinyl acetate (PVAc) and polyvinyl chloride (PVC) over an extensive temperature range. The objective of this study is to investigate the degradation of these polymers in chlorobenzene. The variation of the rate coefficients with temperature is attributed to the change in the kinematic viscosity and vapor pressure.

\section{EXPERIMENTAL}

\section{Ultrasound degradation experiments}

PVAc (prepared by bulk polymerization) and PVC (Aldrich Chemicals) were used for the experiments. The solvent chlorobenzene (S. D. Fine Chemicals) was 
distilled twice and filtered with $0.2 \mu \mathrm{m}$ membrane (Millipore, MA) before use. The amount of $20 \mathrm{~cm}^{3}$ of polymer solution $\left(2 \mathrm{~g} \mathrm{~L}^{-1}\right)$ was taken in a $50 \mathrm{~cm}^{3}$ glass beaker and the beaker was kept in a constant temperature bath $\left( \pm 1^{\circ} \mathrm{C}\right)$. It was ensured that the temperature in the beaker was same as that of the constant temperature bath. The temperature in the reaction vessel was measured at periodic intervals of $10 \mathrm{~min}$ and the temperature was found to be the same. The sonic horn (Vibronics, $25 \mathrm{kHz}$ and $180 \mathrm{~W}$ ) (India) was dipped into the solution of the beaker, and the gap between horn and beaker was closed to reduce any vaporization loss of the solvent. The ultrasonic horn is switched on after the solution attains the bath temperature. Samples were collected at regular time intervals for analysis by gel permeation chromatography (GPC). Many of the experiments were repeated thrice and the standard deviation in the rate coefficients was less than $2 \%$.

\section{GPC analysis}

A two hundred microliter aliquot of these samples was injected in the high performance liquid chromatography (HPLC)-GPC system of Waters, Inc. (MA). The HPLC consists of an isocratic pump, a sample loop, three GPC columns (Waters HR4, HR3, HR0.5) of varying pore sizes, and an online differential refractive index detector. Tetrahydrofuran was used as eluent and it was pumped at a constant flow rate of 1.0 $\mathrm{mL} / \mathrm{min}$. The refractive index is continuously monitored to obtain the chromatograph. The chromatograph is converted to molecular weight distribution by the use of a calibration curve. ${ }^{11}$ The calibration curve was based on polystyrene standards and the molecular weights were converted to PVAc and PVC molecular weights using appropriate $K$ and $\alpha$ values. The initial number average molecular weight and polydispersity of PVAc was 212,000 and 1.12 and that for PVC was 172,000 and 1.3, respectively.

\section{THEORETICAL MODEL}

As discussed earlier, the degradation by ultrasound yields two daughter products of nearly the same chain length. The overall degradation with a rate coefficient of $k$ can thus be represented by

$$
P(x) \rightarrow 2 P(x / 2)
$$

The term $P(x)$ represents a polymer species of chain length $x$, whose molar concentration is represented by $p(x, t)$. No repolymerization of the degraded species was observed in the experiments. This was confirmed by the GPC chromatograph, which showed no molecular weight products higher than the initial distribution.
Continuous distribution kinetics, based on population balances, is used to obtain the degradation rates of polymers. The population balance equation for the above generalized equation is ${ }^{15}$

$$
\begin{aligned}
\frac{\partial p(x, t)}{\partial t}=-k(x) & p(x, t) \\
& +2 \int \propto k\left(x^{\prime}\right) p\left(x^{\prime}, t\right) \delta\left(x-\frac{x^{\prime}}{2}\right) d x^{\prime}
\end{aligned}
$$

The degradation is assumed to be first order with the polymer concentration $p(x, t) .{ }^{16}$ The degradation rate, $k(x)$, is assumed to be of the form $k(x)=k\left(x-x_{1}\right)$, where the $x_{1}$ represents the limiting molecular weight. This ensures that the rate coefficient, $k$, is independent of $x$ and becomes zero when $x=x_{1}$ and no further degradation takes place. Applying the moment operation $\int_{0}^{\infty} x^{(n)}[] d x$, to the above equation yields,

$$
\frac{d p^{(n)}}{d t}=k p^{(n+1)}\left(2^{1-n}-1\right)-k p^{(n)} x_{1}\left(2^{1-n}-1\right)
$$

This equation represents the variation of the polymer MWD moments with time, and solved with the initial conditions.

\section{RESULTS AND DISCUSSION}

The zeroth moment $(n=0)$

$$
\frac{d p^{(0)}}{d t}=k p^{(1)}-k x_{1} p^{(0)}
$$

is solved with the initial condition, $p^{(0)}(t=0)=p_{0}{ }^{(0)}$ to obtain

$$
\left[\frac{\left(p^{(1)}-p_{0}^{(0)} x_{1}\right)}{\left(p^{(1)}-p^{(0)} x_{1}\right)}\right]=e^{k x_{1} t}
$$

In eq. (5), $p^{(0)}$ and $p^{(1)}$ represent the molar and mass concentrations of the polymer. The first moment $(n$ $=1$ ) by eq. (3) yields, $d p^{(1)} / d t=0$, indicating that the polymer mass is conserved. The number average MW, $M_{n}$ is defined as $p^{(1)} / p^{(0)}$, and the above equation reduces to

$$
\ln \left[\frac{\left(x_{1}^{-1}-M_{n 0}^{-1}\right)}{\left(x_{1}^{-1}-M_{n}^{-1}\right)}\right]=\ln H=k x_{1} t
$$

Equation (6) thus represents the variation of $M_{n}$ with time in terms of the initial number average $\mathrm{MW}, M_{n 0}$, and the limiting MW, $x_{1}$. Based on a few experiments conducted for a long time, it was found that the lim- 


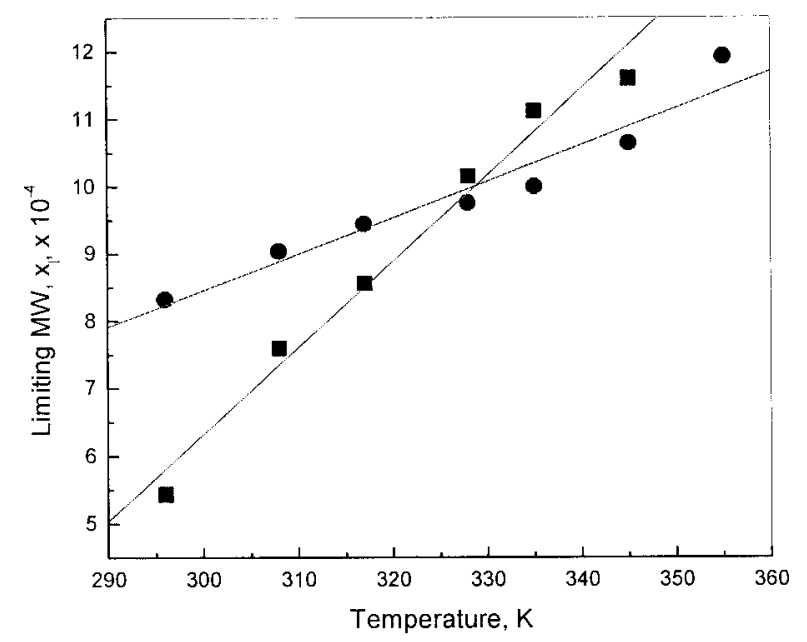

Figure 1 Variation of the limiting $\mathrm{MW}, x_{1}$, with temperature. Legend: (@) PVC and (ם) PVAc.

iting MW is approximately $80 \%$ of the $M_{n}$ at $150 \mathrm{~min}$. The limiting MW for the polymers at all the investigated temperatures were determined and the variation of $x_{1}$ with temperature was nearly linear, as shown in Figure 1. This is consistent with Price and Smith, ${ }^{14}$ who observed a linear variation of the limiting MW with temperature for the degradation of polystyrene in toluene.

Figures 2 and 3 show the variation of $\mathrm{H}$ with time for PVAc and PVC. The semilog plots are linear within experimental error confirming the applicability of eq. (6). The regressed slopes determined at various temperatures gives the product of corresponding rate coefficients and limiting molecular weight. The rate coefficients are thus obtained by dividing the slopes by $x_{1}$. The rate coefficients for PVAc were in the range of

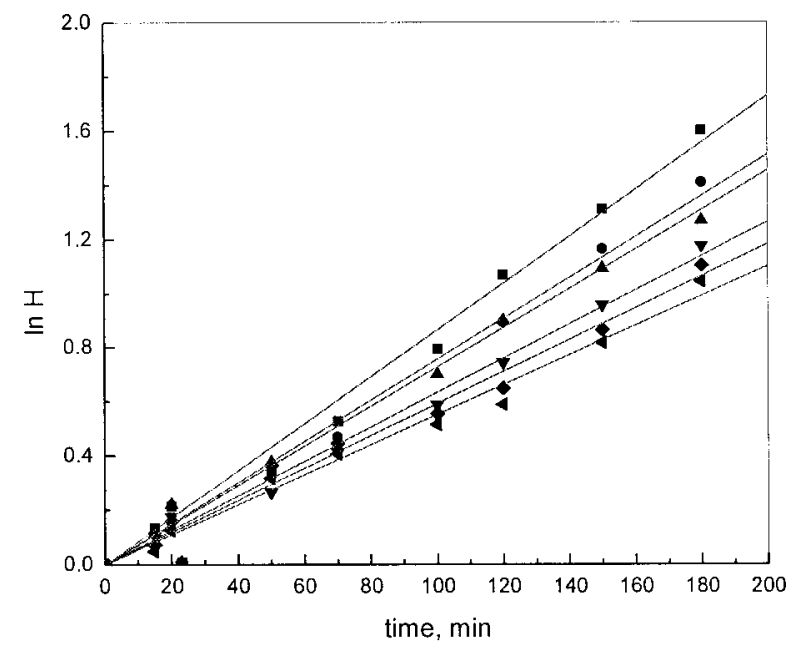

Figure 2 Semilog plot of $\left(x_{l}^{-1}-M_{n 0}^{-1}\right) /\left(x_{l}^{-1}-M_{n}^{-1}\right)$ with time for the ultrasonic degradation of PVAc vs time for PVAc degradation in chlorobenzene at various temperatures. See Figure 3 for legend.

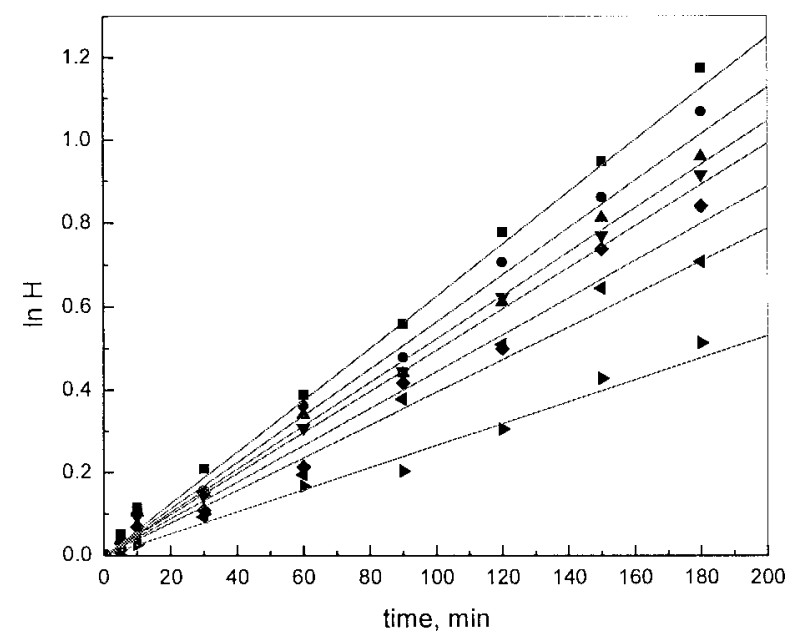

Figure 3 Plot of $\left.\ln \left[\left(x_{l}^{-1}-M_{n 0}^{-1}\right) /\left(x_{l}^{-1}-M_{n}^{-1}\right)\right]\right)$ with time for PVC degradation in chlorobenzene at various tempera-

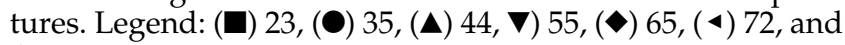
( $82^{\circ} \mathrm{C}$

$1.6 \times 10^{-7}$ to $4.8 \times 10^{-8} \mathrm{~mol} \mathrm{~g}^{-1} \mathrm{~min}^{-1}$ for temperatures of 23 and $72^{\circ} \mathrm{C}$ respectively. Rate coefficients for PVC were from $7.7 \times 10^{-8}$ and $2.3 \times 10^{-8} \mathrm{~mol} \mathrm{~g}^{-1}$ $\mathrm{min}^{-1}$. The rate coefficients for the two polymers indicate that PVAc breaks much more easily than PVC. This may be because the glass transition temperature of PVAc is lower than the glass transition temperature for PVC. It is clear that both polymers (PVAc and PVC) degrade less efficiently at higher temperatures.

Contrary to chemical and thermal reactions, the rate coefficients decrease with increasing temperatures and an Arrhenius plot yields negative activation energies. Negative values of the energy of activation were observed (Figure 4) for both PVAc $(-18.2 \mathrm{~kJ} /$ $\mathrm{mol})$ and PVC $(-17.5 \mathrm{~kJ} / \mathrm{mol})$ similar to the observation of a negative activation energy $(-17.3 \mathrm{~kJ} / \mathrm{mol})$ for the degradation of polystyrene in solution. ${ }^{14}$ The sonic breakage is akin to mechanical breakage in a shear field and thus the calculation of energy of activation does not give any useful information about the process. The decrease in the degradation rate with temperature can be attributed to cavitation. As the temperature of the solution increases, a large quantity of the solvent vapor enters the cavitation bubble during their expansion and exerts a cushioning effect ${ }^{14}$ during the collapse leading to diminishing the intensity of the shock wave. This reduces the jet velocity, ${ }^{10}$ leading to lesser degradation at higher temperatures.

The second phenomenon observed was the sharp change in the degradation rate near the glass transition temperature $\left(T_{g}\right)$. Though this is not clearly evident in Figure 5 for PVC, the change in the degradation rate near $T_{g}$ is clearly seen in the Arrhenius plot (Fig. 4). PVAc $\left(T_{g}=29^{\circ} \mathrm{C}\right)$ shows this sharp drop between 23 and $35^{\circ} \mathrm{C}$ while PVC $\left(T_{g}=79^{\circ} \mathrm{C}\right)$ exhibits this drop near $70^{\circ} \mathrm{C}$. The general explanation that the 


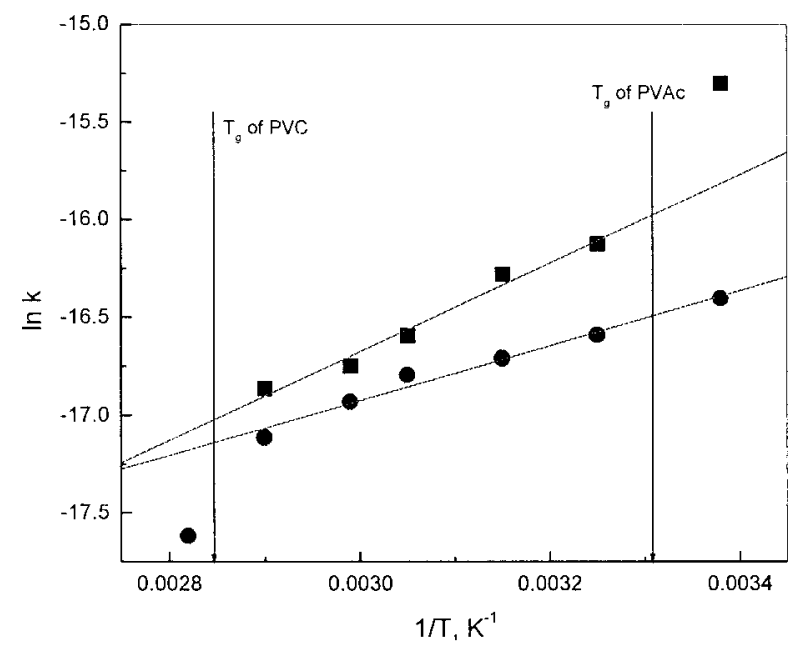

Figure 4 Arrhenius plot of temperature dependence of the rate coefficient, $k$, for PVAc and PVC to determine the activation energies. See Figure 1 for legend.

chains become more flexible ${ }^{17}$ as temperature exceeds $T_{g}$ is applicable for solids but may not be extrapolated to dilute solutions. More experimental data for several polymers covering the temperature range including $T_{g}$ is needed before definite conclusions may be made as to why this phenomena is observed.

Figure 6 shows the effect of vapor pressure on the degradation rate coefficients. As the vapor pressure of the solvent increases, the cavity formed occupies more vapor and thus during collapse it generates microjets of lesser intensity, ${ }^{10}$ leading to lower breakage.

The variation of rate coefficients against kinematic viscosity of the polymer solution (Fig.7) shows an increase of kinematic viscosity leads to increased degradation rates. A better transmission of the shock waves in solution of higher kinematic viscosity could be a probable reason for this higher breakage. ${ }^{11}$ The

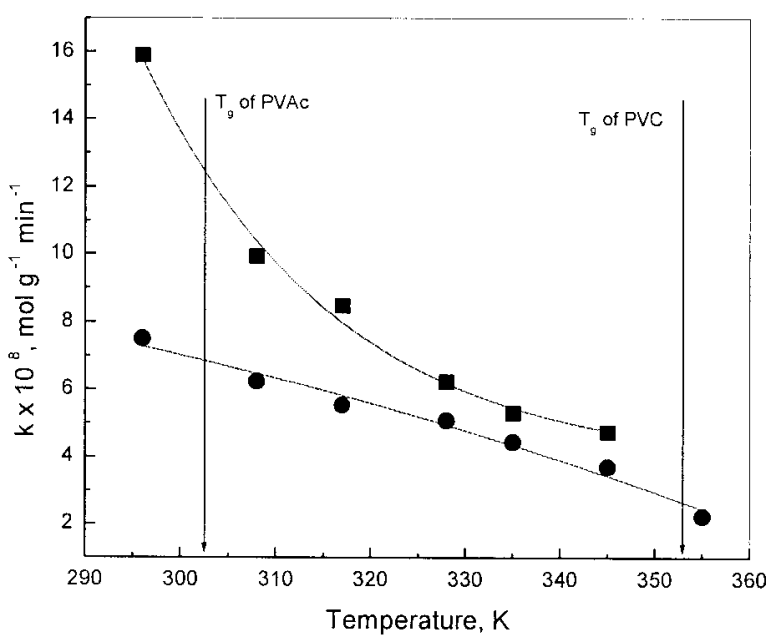

Figure 5 Variation of the degradation rate coefficients with temperature. See Figure 1 for legend.

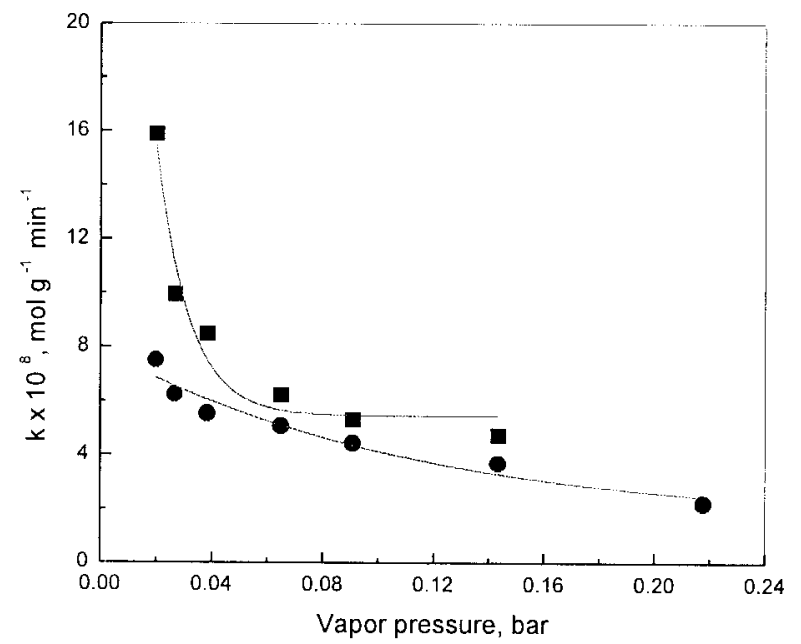

Figure 6 Plot of the degradation rate coefficients of PVAc and PVC with vapor pressures of the solution. See Figure 1 for legend.

variation of the degradation rate coefficients with vapor pressure and kinematic viscosity are also consistent with earlier studies ${ }^{5,11,14}$ that investigated the variation of the degradation rates with these parameters.

\section{CONCLUSION}

The effect of temperature on the ultrasonic degradation of PVC and PVAc was investigated. The experimental results indicated that the degradation rates reduced with increasing temperatures, and could be qualitatively explained by the influence of solvent vapor pressure and kinematic viscosity on cavitation.

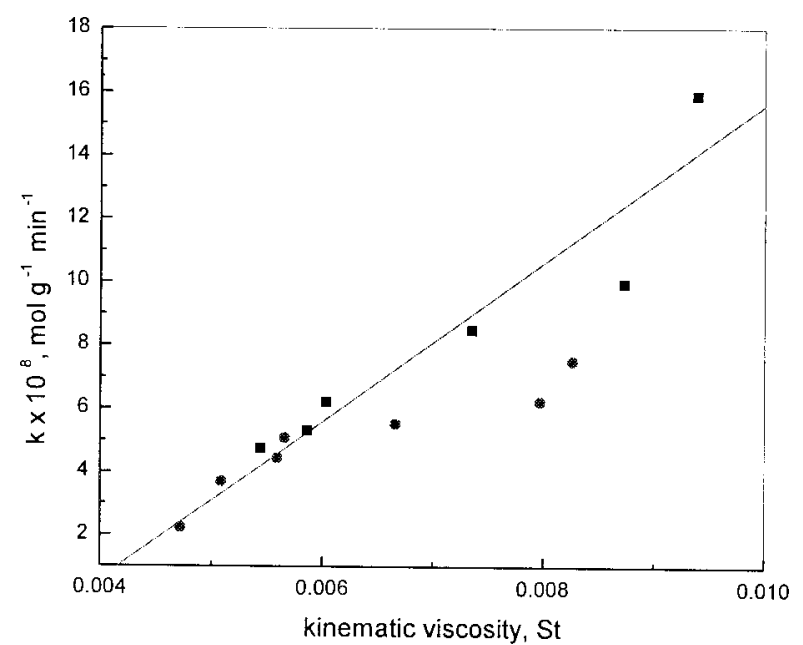

Figure 7 Variation of the degradation rate coefficients with kinematic viscosity of the polymer solution. See Figure 1 for legend. 
The authors wish to thank the Department of Science and Technology, India for financial support.

\section{References}

1. Price, G. J.; Norris, D. J.; West, P. J. Macromolecules 1992, 25, 6447.

2. Cheung, H. M., Gaddam, K. J Appl Polym Sci 2000, 76, 101

3. Ley, S. V.; Low, C. M. R. Ultrasound in Synthesis; SpringerVerlag: New York, 1989.

4. Basedow, A. M.; Ebert, K. H. Adv Polym Sci 1977, 22, 83.

5. Price, G. J. Adv Sonochem 1990, 1, 231.

6. Suslick, K. S. Ultrasound: Its Chemical, Physical and Biological Effects; VCH Publishers:, New York, 1990.
7. Reighley, L. Phil Mag 1917, 34, 94.

8. Thomas, J. R. J Phys Chem 1959, 63, 1725.

9. Hickling, R.; Plesset, M. S. Phys Fluid 1964, 7, 7.

10. Dear, J. P.; Field, J. E. J Fluid Mech 1988, 190, 409.

11. Madras, G.; Chattopadhyay, S. Polym Degrad Stab 2001, 71, 273.

12. Thomas, B. B.; Alexander, W. J. J Polym Sci 1957, 25, 285.

13. Malhotra, S. L. J Macromol Sci, Chem 1982, A17, 601.

14. Price, G. J.; Smith, P. F. Polymer 1993, 43, 4111.

15. McCoy, B. J.; Madras, G. AIChE J 1997, 43, 802.

16. Glynn, P. A.; Van der Hoff, Reilly, P. M. J Macromol Sci 1972, A6, 1653.

17. Malhotra, S. L. J Macromol Sci, Chem 1983, A18, 1055. 